\title{
РАЗРАБОТКА КОМПЬЮТЕРНОЙ МОДЕЛИ ТЕПЛОВОГО НАСОСА С ПОСТОЯННОЙ ЧАСТОТОЙ ВРАЩЕНИЯ СПИРАЛИ КОМПРЕССОРА
}

\author{
Д.В. Тимофеев, Е.Г. Малявина
}

Национальный исследовательский Московский государственный строительный университет (НИУ МГСУ), 129337, г. Москва, Ярославское шоссе, д. 26

\begin{abstract}
АННОТАЦИЯ. Тепловые насосы применяются для отопления зданий и сооружений. Определение теплотехнических характеристик теплового насоса в режимах, отличных от номинального, связано с определенными трудностями, поскольку в холодном климате России эти характеристики, указанные производителями в соответствии с требованиями стандартов, будут отличаться в худшую сторону.

В работе были поставлены следующие цели: компьютерное моделирование теплового насоса вода-вода или вода-гликоль и его представление в виде системы моделей двух теплообменников и спирального компрессора; проведение численных расчетов геотермальной системы теплоснабжения и оценка ее эффективности при разных конфигурациях и режимах работы с помощью компьютерного моделирования.

Программный пакет для компьютерного моделирования написан на языке Scala, для получения параметров модели из данных о работе компрессора и теплового насоса используются алгоритмы Нелдера-Мида и Левенберга-Марквардта, для одномерной минимизации применяется метод золотых сечений.

Полученная модель состоит из отдельных моделей компрессора, теплообменников испарителя и конденсатора. Модель компрессора учитывает полезный перегрев хладагента, работу на различных хладагентах. Модели теплообменников выполнены упрощенно. Параметры модели вычисляются по данным, указанным производителями оборудования тепловых насосов и компрессоров.

Модель аппроксимирует данные производителя оборудования, поэтому может использоваться как элемент геотермальной системы в программах энергетического моделирования. При помощи модели можно также определить реальные характеристики работы теплового насоса на режимах, отличных от номинального, и при использовании разных хладагентов. Еще одно приложение полученных результатов - выявление недобросовестных производителей, указывающих фриктивные данные в своих каталогах.
\end{abstract}

КЛЮЧЕВЫЕ СЛОВА: тепловой насос, спиральный компрессор, параметры хладагента, геотермальная энергетика, Scala, компьютерное моделирование, энергетическое моделирование

дЛя цИтИРОВАнИЯ: Тимофеев Д.В., Малявина Е.Г. Разработка компьютерной модели теплового насоса с постоянной частотой вращения спирали компрессора // Вестник МГСУ. 2017. Т. 12. Вып. 4 (103). С. 437-445. DOI: 10.22227/1997-0935.2017.4.437-445

\section{COMPUTER MODEL OF HEAT PUMP WITH A CONSTANT ROTATION FREQUENCY OF A SCROLL COMPRESSOR SPIRAL}

\author{
D.V. Timofeev, E.G. Malyavina \\ Moscow State University of Civil Engineering (National Research University) (MGSU), \\ 26 Yaroslavskoe shosse, Moscow, Russian Federation, 129337
}

ABSTRACT. Heat pumps are used for heating buildings and structures. Determination of thermal characteristics of a heat pump in modes other than the nominal ones is associated with certain difficulties, since, in the cold climate of Russia, these characteristics, indicated by the manufacturers in accordance with the requirements of the standards, will differ for the worse.

In study, the following purposes were set: computer simulation of the water-water or water-glycol heat pump and its representation in the form of a simulation system of two heat exchangers and a scroll compressor; conducting numerical calculations of the geothermal heat supply system, estimating its efficiency both under different configurations and operate modes using computer simulation.

The software package for computer simulation is written in the language of Scala, both Nelder-Mead and LevenbergMarquardt algorithms are used to obtain model parameters from the compressor and heat pump data, the method of Gold Cross Section is used for one-dimensional minimization. The presented simplified model for heat pump parameter estimation aims to selection of pump size and simulation its operation during year. While the scroll compressor model is made to be comprehensive, the other parts of heat pump model were simplified.

The resulting model consists of individual compressor models, evaporator heat exchangers, and refrigerator. The compressor model takes into account the useful overheating of the refrigerant, work on various refrigerants. Models of heat exchangers are simplified. The model parameters are calculated from the data specified by the manufacturers of heat pump and compressor equipment.

The model approximates the data of the equipment manufacturer and, therefore, can be used as an element of the geothermal system in energy modeling programs. Using the model, it is also possible to determine the actual characteristics of the heat pump operation in modes other than the nominal one and with the use of different refrigerants. Another application of the obtained results is the identification of unscrupulous manufacturers indicating fictitious data in their catalogs. Obtained 
model can be extended by including other refrigerants, variable compressor motor rotation frequency, and more precise implementation of the other heat pump parts.

KEY WORDS: heat pump, compressor spiral, refrigerant parameters, geothermal energy, scala, computer simulation, energy simulation

FOR CITATION: Timofeev D.V., Malyavina E.G. Razrabotka komp'yuternoy modeli teplovogo nasosa s postoyannoy chastotoy vrashcheniya spirali kompressora [Computer Model of Heat Pump with a Constant Rotation Frequency of Scroll Compressor Spiral]. Vestnik MGSU [Proceedings of Moscow State University of Civil Engineering]. 2017, vol. 12, issue 4 (103), pp. 437-445. (In Russian) DOI: 10.22227/1997-0935.2017.4.437-445

Существует проблема в определении теплотехнических характеристик теплового насоса на режимах, отличных от номинального. При понижении температуры на испарителе или повышении температуры на конденсаторе теплового насоса сильно уменьшается его теплопроизводительность или потребляемая электрическая мощность - в зависимости от типа используемого компрессора. Согласно существующим международным нормам ${ }^{1}$, производители могут указывать значения на одном или нескольких номинальных режимах, но в холодном климате России эти характеристики почти всегда будут хуже. Помимо определения проектных характеристик теплового насоса, т.е. ответа на вопрос, будет ли тепловой насос работать в самых тяжелых погодных условиях, важно знать информацию о его работе в течение года, т.е. какую отопительную нагрузку здания он покроет, сколько холода при этом придется ассимилировать грунтовому контуру, сколько суммарно электроэнергии на его работу будет затрачено и т.п.

Существующие модели тепловых насосов условно можно разделить на три группы:

1. Известны конструктивные характеристики всех комплектующих теплового насоса и их поведение в различных ситуациях, например во время включения защиты от замораживания. Моделью теплового насоса в таком случае будет система моделей его комплектующих [1] (determenistic model определительная модель);

2. Конструкция самого теплового насоса представляет собой «черный ящик». В ходе расчета применяются формулы «пересчета», использующие в качестве параметров теплотехнические характеристики теплового насоса из каталогов производителя на номинальном режиме [2-4] (equation fit model модель на аппроксимирующих формулах);

3. Известны основные теплотехнические характеристики теплового насоса на номинальном режиме и некоторые конструктивные, предоставляемые производителями в каталогах. Вычисляются недостающие конструктивные характеристики, на их основании строится упрощенная модель теплового насоса [5-7] (parameter estimation model - модель на основе вычисления параметров).

${ }^{1}$ EN 14511-1, 2, 3, 4. Air conditioners, liquid chilling packages and heat pumps with electricaly driven compressors for space heating and cooling, 2013.
Модели первой группы используются при конструировании самого теплового насоса. Они позволяют сравнить характеристики теплового насоса с разными комплектующими. В этом заинтересованы, главным образом, производители оборудования. Модели второй и третьей групп используются проектировщиками или специалистами по энергетическому моделированию для получения информации об эффективности или вообще о возможности применения теплового насоса в заданных климатических условиях. Модели второй группы встречаются в литературе все реже и реже, а представленную ниже модель можно отнести к третьей группе.

Параметрами модели теплового насоса (рис. 1) являются произведения коэффициента теплопередачи и их площади: для испарителя $k_{0} F_{0}$, и конденсатора $k_{1} F_{1}, \mathrm{BT} /{ }^{\circ} \mathrm{C}$. В качестве допущения эти произведения принимаются постоянными при любых расходах теплоносителя в грунтовом контуре и в системе отопления. Они не вводятся пользователем, а вычисляются на основе теплотехнических данных для одного или нескольких номинальных режимов, представленных производителем. Варианты пересчета этих коэффициентов приведены в статьях $[5,8]$. Третьим параметром модели является вложенная модель компрессора. Рассматриваемая модель разработана для наиболее распространенного в настоящее время спирального компрессора и выполнена на основе работ [9, 10].

Параметрами модели компрессора являются:

- коэффициент теплопередачи участка трубы от испарителя до компрессора (всасывающего патрубка), умноженного на площадь $k F, \mathrm{BT} /{ }^{\circ} \mathrm{C}$;

- коэффициенты для нахождения фиктивной температуры стенки всасывающего патрубка $T_{w}$ : $a_{w}, b_{w}, c_{w}$;

- рабочий объем компрессора $V_{s}, \mathrm{~cm}^{3}$;

- скорость вращения компрессора $N$, об./c;

- коэффициенты псевдоизоэнтропной эффективности $a, b$;

- отношение рабочего объема компрессора к объему хладагента при промежуточном давлении IP (intermediate pressure): $V_{s} / V_{e x}$.

При этом в качестве исходных данных требуется только значение объемного расхода теплоносителя и потребляемой мощности при номинальных значениях температуры на испарителе и конденсаторе, значение рабочего объема компрессора и скорости вращения его спирали. Их можно найти 


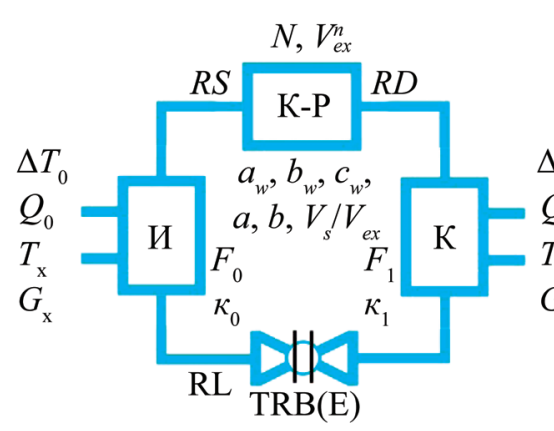

$a$

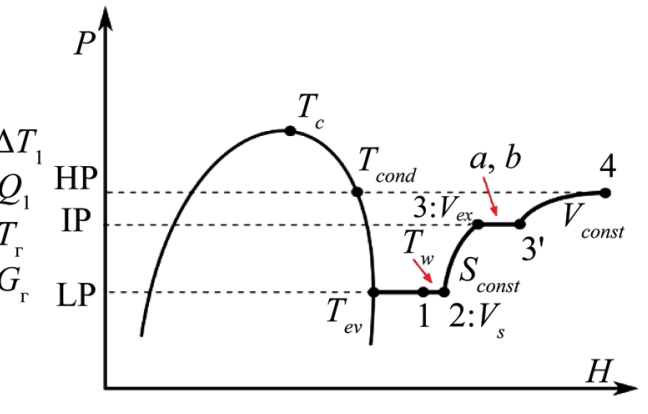

$\sigma$

Рис. 1. Параметры модели теплового насоса и компрессора: $a$ - обозначения на схеме теплового насоса (внутри контура теплового насоса параметры, определяющиеся расчетом, снаружи - задающиеся перед расчетом и в процессе моделирования); $\sigma$ процесс сжатия хладагента в компрессоре согласно описанию [10] с указанием влияния параметров на конкретный процесс или точку

среди технической информации, представляемой производителем. Все остальные данные определяются методами минимизации погрешностей между рассчитанными величинами и представленными производителем.

В работе была принята модель [9, 10], которая является упрощением модели [11] и использует ее как основу. В ней частота вращения компрессора принимается постоянной и определяются мощность, объемный расход и теплота полезного перегрева при помощи моделирования изопроцессов, которые происходят с хладагентом в процессе его сжатия (см. рис. 1). Число необходимых конструктивных характеристик в модели было сведено к минимуму. Сжатие хладона в компрессоре можно представить как последовательность следующих процессов:

- нагрев хладагента на линии всасывания у входа в компрессор (полезный перегрев), получение объема $V_{s}$ (тт. $\left.1-2\right)$;

- изоэнтропное сжатие хладагента до объема $V_{e x}=V_{s} /\left(V_{s}^{n} / V_{e x}^{n}\right)$, определение промежуточного давления IP (тт. 2 - 3);

- получение реального объема хладагента в конце предыдущего процесса при том же давлении с учетом коэффициента неизоэнтропности (3) (тт. 3-3');

- изохорный процесс до давления на конденсаторе HP (high pressure) (тт. 3' - 4).

Мощность компрессора определяется по формуле

$$
P_{\text {comp }}=\frac{q_{m}\left(h_{3}-h_{2}\right)}{\eta_{\text {pseudo-iso-s }}}+(\mathrm{HP}-\mathrm{IP}) q_{m} v_{3 \text { real }},
$$

где $q_{m}$ - массовый расход хладагента, кг/с; $h_{2}$, $h_{3}$ - энтальпии в точках 2 и 3 , Дж/кг; $\eta_{\text {pseudo-iso-s }}-$ коэффициент псевдоизоэнтропной эффективности; $v_{\text {зreal }}$ - удельный объем хладагента, $\mathbf{m}^{3} / \kappa г$.

Подробности определения параметров в формуле (1) представлены в статье [9], а здесь остановимся на тех моментах, которые там были недостаточно освещены.

Массовый расход хладагента при температурах испарителя и конденсатора определяется при параметрах хладагента на входе в компрессор после того, как хладон прошел испаритель и дополнительно перегрелся по ходу к компрессору. Этот перегрев (полезный перегрев) определяется из формулы теплопередачи через стенку участка трубы от испарителя до компрессора при помощи введения фиктивной температуры на наружной поверхности стенки этой трубы, которая находится как

$$
T_{w}=a_{w}+b_{w} T_{e v}+c_{w} T_{\text {cond }} .
$$

Фиктивной она называется потому, что вместе с величиной $k F$ и коэффициентами в формуле ее значение определяется при помощи минимизации погрешности между известным из каталога расходом хладагента на номинальном режиме и рассчитанным [10], и множество пар значений может подойти. При этом значение $T_{w}$ не обязательно должно соответствовать реальной температуре стенки всасывающего патрубка. Операция осуществлялась при помощи алгоритма ЛевенбергаМарквардта с набором неизвестных точек [12]. Для получения корректного результата требуется около 12 точек. Еще одна формула с неизвестными параметрами определяет коэффициент неизоэнтропности сжатия:

$$
\eta_{\text {pseudo-iso-s }}=a \frac{\mathrm{IP}}{\mathrm{LP}}+b
$$

Значение IP определяется при объеме $V_{e x}$, который находится при помощи коэффициента $V_{s} / V_{e x}$. $a, b, V_{s} / V_{e x}-$ неизвестные параметры модели, LP - область низкого давления (low pressure). Их значения получаются в процессе минимизации погрешности между рассчитанными и заданными мощностями компрессора на номинальном режиме. Так же, как и в предыдущем случае, требуется около 12 точек для получения точных результатов.

Теплота, которую можно получить при заданных температурах хладагента на испарителе и конденсаторе, определяется значениями температуры перегрева и переохлаждения на основе теплового баланса теплового насоса: 


$$
Q_{\text {cond }}=Q_{e v}+P_{\text {comp }}+Q_{\text {sh }},
$$

где $Q_{\text {cond }}$ - теплота, отданная на конденсаторе, Вт; $Q_{e v}$ - теплота, поглощенная испарителем, Вт; $P_{\text {comp }}$ - мощность компрессора, Вт; $Q_{s h}$ - теплота полезного перегрева хладона на входе в компресcop, Вт.

Помимо модели компрессора, в модель теплового насоса входит упрощенное описание испарителя и конденсатора, которые имеют параметр $k_{i} F_{i}$, $\mathrm{B} \mathrm{T} / \mathrm{K} ; i=0 \ldots 1$. Формула расчета количества теплоты, переданной теплопередачей между теплоносителем и хладагентом на испарителе или конденсаторе $Q$, Вт, записывается как

$$
Q=k_{i} F_{i} \frac{t_{o}-t_{e}}{\ln \left(\frac{t_{r}-t_{e}}{t_{r}-t_{o}}\right)},
$$

где $t_{e}, t_{o}$ - температуры теплоносителя на входе и на выходе из теплового насоса на испарителе или конденсаторе, ${ }^{\circ} \mathrm{C} ; t_{r}$ - температура хладагента на испарителе и конденсаторе, ${ }^{\circ} \mathrm{C}$.

Допущение постоянства коэффициентов $k_{i} F_{i}$ при изменении расхода хладагента и теплоносителя в дальнейшем можно будет исправить. При этом принято, что электронный расширительный вентиль всегда может сбросить давление до необходимого. Значения $k_{i} F_{i}$ находятся при помощи минимизации погрешностей расчетных и фактических данных нагрузок на первичном или вторичном контурах и мощности по данным производителя (обычно они предоставляют два режима, что вполне достаточно). Для минимизации использовался алгоритм Нелдера-Мида [12], который в данном случае сходится к одному минимуму. Алгоритм нахождения характеристик показан на рис. 2.
Исходными данными в каждой итерации являются температура на улице, его входные температуры и расходы. По температуре наружного воздуха, согласно заданному графику ищется требуемая температура на выходе из теплового насоса $t_{o 1}^{\prime}$.

Зная требуемую температуру на выходе из теплового насоса, можно найти теплоту $Q_{1}$, которую нужно сообщить теплоносителю, чтобы его температура достигла значения $t_{o 1}^{\prime}$, на основе которой проверяется, не замерзнет ли теплоноситель на первичном контуре, если ему сообщить теплоту $Q_{1}$. Тогда выдается рекомендуемое значение количества теплоты, которое может сообщить теплообменник в контуре здания $Q_{1}^{f}$. Зная требуемое количество теплоты, входные температуры и расход теплоносителя, определяют температуры хладагента на испарителе и конденсаторе, симулируют работу компрессора и теплообменников и выдают рабочие выходные характеристики теплового насоса: выходные температуры $t_{o 0}, t_{o 1}$, мощность привода компрессора $P_{\text {сотр }}$, и количества теплоты $Q_{1}, Q_{0}$, переданные и забранные у каждого контура.

Для применения вышеописанной модели компрессора необходимо получать свойства хладагента на линии насыщения пара, линии конденсации пара и в состоянии перегретого пара. В работе [9] для этого используется программа REFPROP, на основе которой получается полиноминальная зависимость от значений температуры испарителя и конденсатора. В нашем случае такой подход неудобен. Вызывать функции прикладного программного интерфейса REFPROP, написанного на языке Фортран из JVM, на котором ведется разработка, пришлось бы через язык $\mathrm{C}$, что увеличило бы техническую сложность приложения и время вызова каждого метода REFPROP. Гибкость программы при этом снизилась бы.

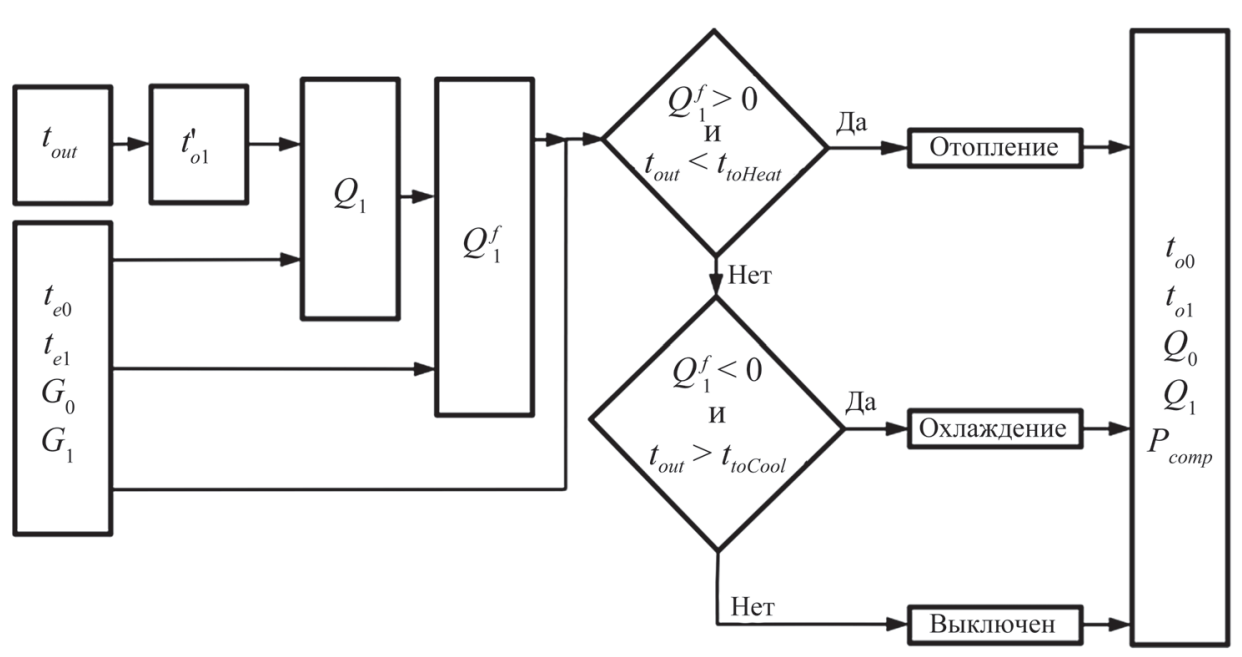

Рис. 2. Блок-схема, описывающая работу теплового насоса 
Кроме того, сведение модели к полиноминальной формуле, увеличивая производительность программы, уменьшает ее гибкость. К примеру, при учете переменной частоты вращения компрессора в работе [10] придется менять конечную формулу, добавляя в нее частоту вращения спирали компрессора, нам же будет достаточно выяснить характер изменения этой частоты. Поэтому разработана и запрограммирована модель, рассчитывающая нужные параметры хладагента. Для аппроксимации параметров хладона на линии смены фаз жидкости была применена сплайновая интерполяция на основе монотонных сплайнов [13]. Использование именно этого типа сплайнов было продиктовано известностью критических параметров, которые являются экстремумами параметров хладона. Приняв значения критических параметров в одной из точек сплайна, можно быть уверенным, что он точно повторит геометрическую форму линии насыщения и конденсации. Параметры теплоносителя в точках на линии насыщения и конденсации пара были взяты по данным производителей $[14,15]$, которые тесно связаны с базой REFPROP [16]. Для аппроксимации давления, объема и температуры перегретого пара хладагента была принята модель новых уравнений состояния (NEoS - New Equation of State) [17]:

$$
P=\frac{R T}{V-b}-\frac{a(T)}{V^{2}+u b V+w b^{2}},
$$

где $R$ - универсальная газовая постоянная, Дж/(моль $\cdot \mathrm{K}) ; T$ - температура, K; $V$ - молярный объем, м³/моль; $b, u, w$ - коэффициенты, процедура нахождения которых описана в статье [17]; $a(T)-$ функция Матиаса-Коупмана, записывающаяся как

$$
a(T)=a_{c} \alpha(T),
$$

где

$$
\alpha(T)=\left[\begin{array}{l}
1+m_{1}\left(1-\sqrt{\frac{T}{T_{c}}}\right)+ \\
+m_{2}\left(1-\sqrt{\frac{T}{T_{c}}}\right)^{2}+m_{3}\left(1-\sqrt{\frac{T}{T_{c}}}\right)^{3}
\end{array}\right]^{2},
$$

если $T<T_{\mathrm{c}}$; а

$$
\alpha(T)=\left[1+m_{1}\left(1-\sqrt{\frac{T}{T_{c}}}\right)\right]^{2},
$$

если $T>T_{\mathrm{c}}$. Здесь $T_{c}$ критическая температура пара хладагента (см. рис. 1); $m_{i}$ - коэффициенты, значения которых вычисляются согласно [17].

Как видно из формулы, давление можно вычислить напрямую, объем рассчитывается аналитически путем решения кубического уравнения ${ }^{2}$, а температура определяется численно путем минимизации разности между заданным и получен-

\footnotetext{
${ }^{2}$ Стоит отметить, что в формуле для кубического уравнения (6) в работе [17] содержится ошибка: перед многочленом первой степени должен стоять знак «-», а не «+».
}

ным значениями давления по формуле . Формула применима для чистых жидкостей, а параметры смесей в статье [17] предлагается вычислять на основе значений ее компонентов, приняв одно из правил смешивания и предварительно вычислив коэффициенты бинарного взаимодействия компонентов $k_{i j}[18]$. Однако для хладона R410a, при котором будет работать тепловой насос, прямое применение формулы (6) дает результаты даже лучшие, чем для одной из его составляющих, (рис. 3,4$)$ поэтому характеристики указанного хладона вычислялись прямо по формулам NEoS. Значения энтальпии и энтропии рассчитывались при помощи формул теплоемкости для идеального газа чистых веществ, представляющих из себя многочлен четвертой степени [19]. Энтальпия для идеального газа, Дж/моль, определялась как

$$
\begin{aligned}
H^{i g}(T, P)= & H(298,15,101325)+ \\
& +\int_{298,15}^{T} C_{p}(T) d T,
\end{aligned}
$$

где $C_{p}$ - изобарная теплоемкость идеального газа, Дж/(моль $\left.{ }^{\circ} \mathrm{C}\right)$, которая пересчитывалась при помощи функций отклонения (табл. 6-4 из работы [18]) для реальных газов.

Для смеси R410a пришлось использовать дополнительно правило смешивания ее компонентов:

$$
Q_{m}=\sum_{i=1}^{n} \sum_{j=1}^{n} y_{i} y_{j} Q_{i j}
$$

где $Q_{m}$ - результирующий параметр; $y$ - молярная фракция данного компонента; $Q_{i j}$ - правило соединения компонентов.

Были проанализированы различные правила соединения, и среднее гармоническое было принято для расчетов:

$$
Q_{i j}=\frac{2}{\frac{1}{Q_{i i}}+\frac{1}{Q_{j j}}}\left(1-k_{i j}\right),
$$

где $Q_{i i}, Q_{i j}$ - параметры компонентов; $k_{i j}-$ коэффициент бинарного взаимодействия, который рассчитывался при помощи минимизации погрешностей между значениями из программ $[14,15]$ и вычисленными.

Энтропия хладагента, Дж/(моль · К), находилась по формуле

$$
\begin{aligned}
S(T, V)= & S\left(T^{0}, V^{0}\right)+ \\
& +\frac{2\left(H(T, V)-H\left(T^{0}, V^{0}\right)\right)}{T+T^{0}}
\end{aligned}
$$

где $T, T^{0}$ - температуры в искомой точке и при том же давлении на линии насыщения соответственно, К; $V, V^{0}$ - молярные объемы в искомой точке и на линии насыщения соответственно, м $^{3}$ ммоль 


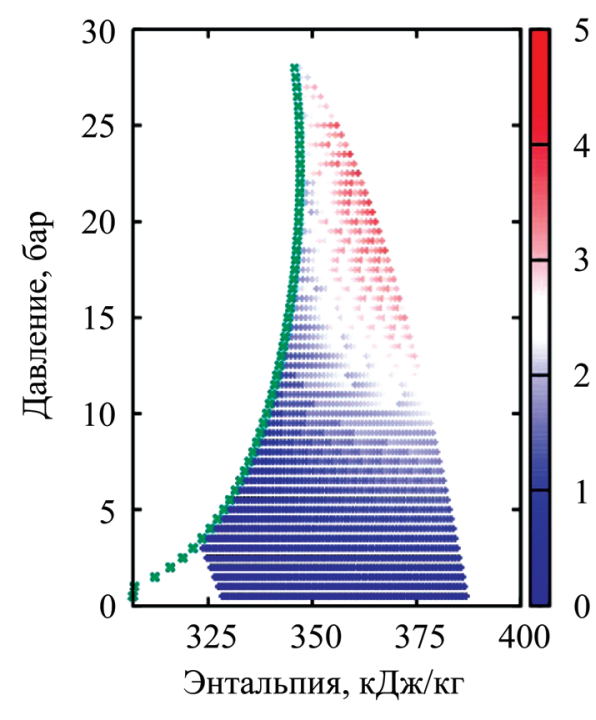

$a$

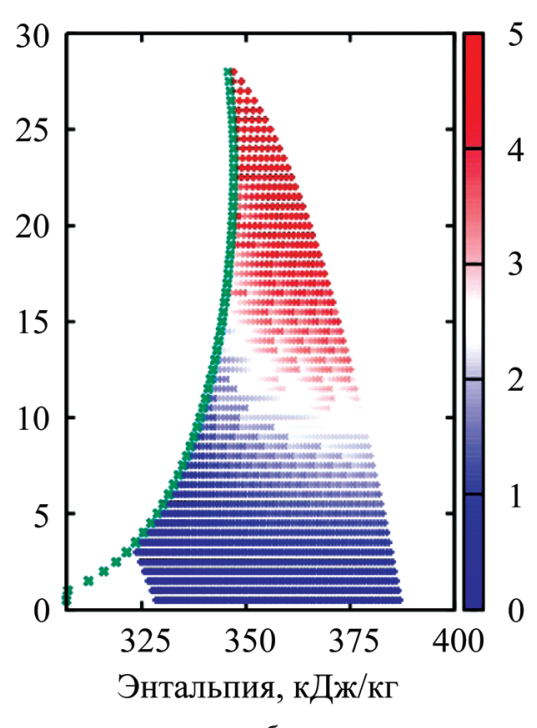

б

Рис. 3. Погрешность на $p$ - $H$-диаграмме в определении давления ( $a$ ) и удельного объема (б) по формулам NEoS для чистой жидкости R125 в сравнении с данными производителя, созданными на основе REFPROP [16] (на цветовой шкале справа — относительная погрешность, \%; × - линия насыщения пара)

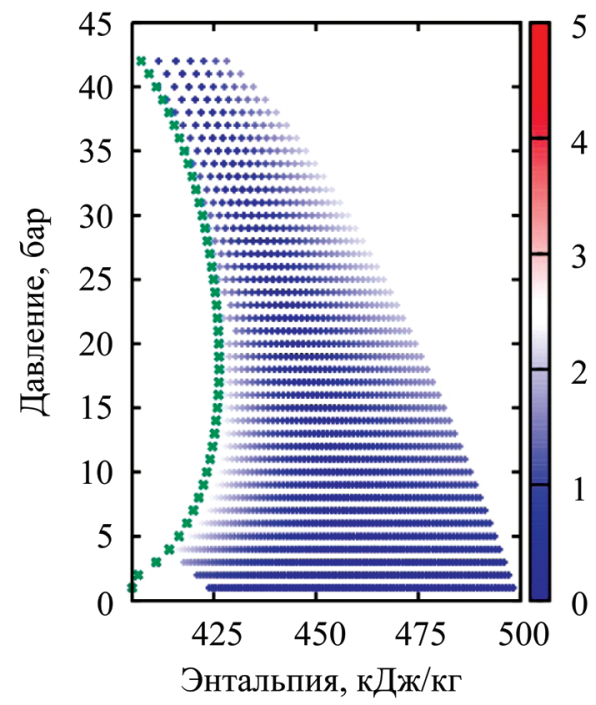

$a$

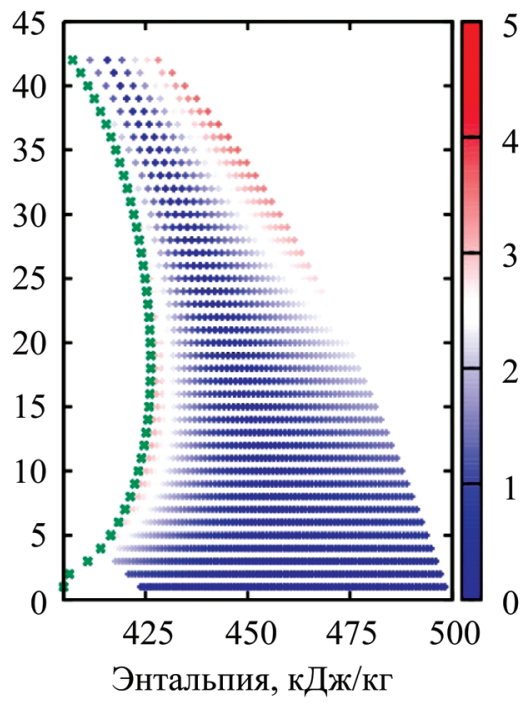

б

Рис. 4. Погрешность в определении давления (a) и относительного объема (б) по формулам NEoS для cмеси R410a, представленной как чистая жидкость, в сравнении с данными производителя (на цветовой шкале справа - относительная погрешность, \%; × — линия насыщения пара)

Для смешивания использовались те же правила смешивания и соединения. Результирующие формулы для вычисления коэффициентов бинарного взаимодействия представлены в таблице.

При помощи модели выполним расчет теплопроизводительности и мощности теплового насоса со следующими исходными характеристиками точек:

- 24300 Вт тепловой нагрузки, 8 кВт мощности, температура на испарителе $0{ }^{\circ} \mathrm{C}$, температура на конденсаторе $35^{\circ} \mathrm{C}$;

- 24200 Вт тепловой нагрузки, 6,8 кВт мощности, температура на испарителе $0{ }^{\circ} \mathrm{C}$, температура на конденсаторе $50^{\circ} \mathrm{C}$;
- компрессор с $V_{s}=88 \mathrm{~cm}^{3}, N=2900$ об/мин, расход хладагента 435 кг/ч, мощность компрессора 5,575 кВт при $T_{e v}=273 \mathrm{~K}, T_{\text {cond }}=313 \mathrm{~K}$.

Результаты представлены на рис. 6, 7.

Для принятого теплового насоса линии изменения нагрузки и мощности изменяются почти линейно, что необязательно для других моделей. На рис. 6 видна характерная для спиральных компрессоров картина [5]: в зависимости от температуры теплоносителя грунтового контура количество производимой теплоты резко падает, тогда как мощность практически не изменяется. Это значит, что проектировщикам, подбирающим тепловой насос, нужно учитывать падение производительности теплового 
Результирующие формулы для вычисления коэффициентов бинарного взаимодействия

\begin{tabular}{l|l}
\hline \multicolumn{1}{c|}{ Для определения } & \multicolumn{1}{c}{$k_{i j}$, при $V, \mathrm{~cm}^{3} /$ кг, и $T, \mathrm{~K}$} \\
\hline энтальпии $H$ & $0,1005-1,4086 \cdot 10^{-7} V-4,7768 \cdot 10^{-10} T$ \\
\hline энтропии $S$ & $-0,075+0,0325 V-0,7111 T$
\end{tabular}
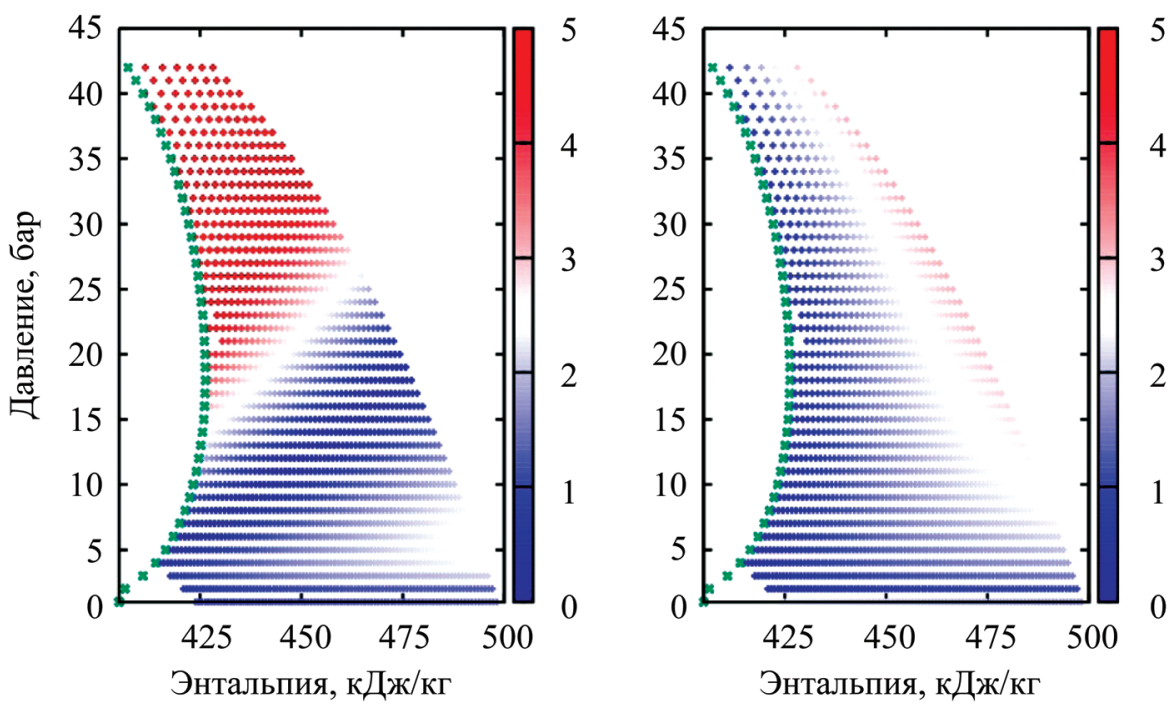

$a \sigma$

Рис. 5. Погрешность в определении: $a$ - энтальпии; $\sigma$ - относительного объема по формулам NEoS для смеси R410a, представленной как чистая жидкость, в сравнении с данными производителя (на цветовой шкале справа — относительная погрешность, \%; × — линия насыщения пара)

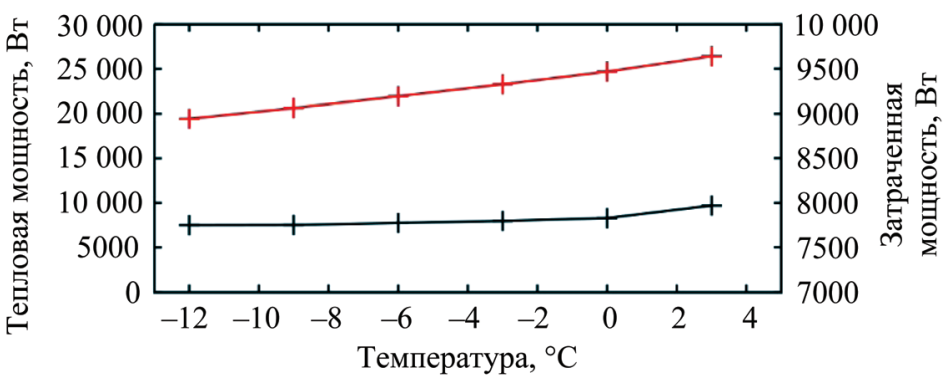

Рис. 6. Работа теплового насоса при температуре входящей жидкости из системы отопления $40{ }^{\circ} \mathrm{C}$, различной температуре входящей жидкости из грунтового контура (по оси $x$ ) и при постоянном расходе теплоносителя: - - теплота, переданная зданию (по левой шкале $y$ ), Вт; - — затраченная мощность теплового насоса (по правой оси $y$ ), Вт

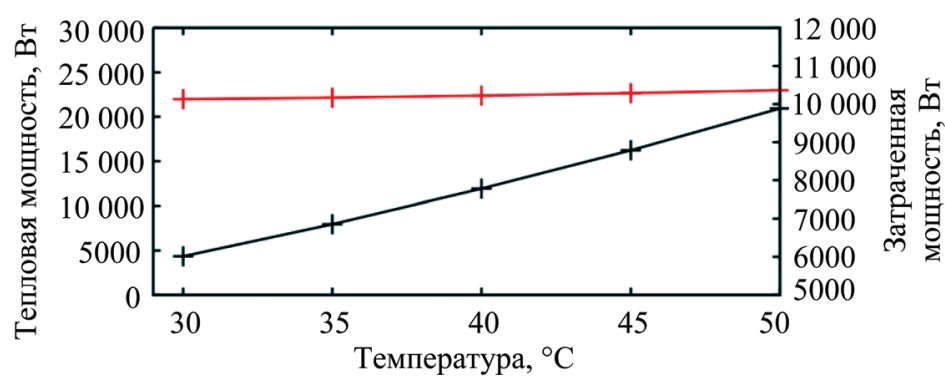

Рис. 7. Работа теплового насоса при температуре входящей жидкости из грунтового контура $0{ }^{\circ} \mathrm{C}$, различной температуре входящей жидкости из системы отопления (по оси $x$ ) и постоянном расходе теплоносителя: - — теплота, переданная зданию (по левой шкале $y$ ), Вт; —— затраченная мощность теплового насоса (по правой шкале $y$ ), Вт 
насоса от температуры грунтового контура. Потребляемая мощность агрегата при этом увеличится при частичной загрузке. Для уменьшения же потребляемой мощности при частичной загрузке лучше закладывать несколько тепловых насосов [20] или устанавливать тепловой насос с переменной частотой вращения компрессора. На рис. 7 изменяется температура системы отопления: произведенная теплота почти не изменяется, а мощность на графике изменяется в два раза. Поэтому нельзя принимать мощность при температуре в системе отопления на входе в тепловой насос меньше проектной.

Разработанная модель позволяет определить теплотехнические параметры теплового насоса в различных температурных условиях. Дальнейшим направлением работы мы видим соединение модели грунтового теплообменника [21] и модели теплового насоса.

\section{ЛИТЕРАТУРА}

1. Chen Y., Halm N.P., Groll E.A. et al. Mathematical modeling of scroll compressors - part I: compression process modeling // International Journal of Refrigeration. 2002. Vol. 25. No. 6. Pp. 731-750.

2. Дуванов С.А., Ильин А.К. Метод моделирования работы тепловых насосов // Вестник Астраханского государственного технического университета. 2005. Вып. 2 (25) C. 66-70.

3. Allien J.J., Hamilton J.F. Steady-state reciprocating water chiller models // ASHRAE Transactions. 1983. 89 (2A). Pp. 398-407.

4. Huchtemann K., Dirk M. Advanced simulation methods for heat pump systems // Proceedings of the 7th Modelica Conference. Como, Italy. 2009, Sep 20-22. Pp. 798-803.

5. Kinab E., Marchio D., Riviere P. et al. Reversible heat pump model for seasonal performance optimizations // Energy and Buildings. 2010. No. 42. Pp. 2269-2280.

6. Jin H., Spitler J. A parameter estimation based model of water to water heat pumps for use in energy calculation programs // ASHRAE Transactions. 2002. 108 (1). Pp. 3-17.

7. Zakula T. Heat pump simulation model and optimal variable-speed control for a wide range of cooling conditions : master of science in building technology thesis. Massachusetts Institute of Technology, 2010.

8. Kavanaugh S., Rafferty K. Geothermal heating and cooling. Design of Ground-Source Heat Pump systems. Atlanta : ASHRAE, 2014. $420 \mathrm{p}$.

9. Duprez M.-E., Dumont E., Frere M. Modeling of reciprocating and scroll compressors // International journal of refrigeration. 2007. No. 30. Pp. 873-886.

10. Duprez M.-E., Dumont E., Frere M. Modeling of scroll compressors - Improvements // Internacional journal of refrigeration. 2010. No 33. Pp. 721-728.

11. Winandy E., Saavedra C., Lebrun J. Experimental analysis and simplified modeling of a hermetic scroll refrigeration compressor // Applied Thermal Engeneering. 2001. Vol. 22. Pp. 107-120.

Поступила в редакиию в августе 2016 г.

Принята в доработанном виде в октябре 2016 г.

Одобрена для публикации в феврале 2017 г.
12. Scalaopt. Scala numerical optimization library // Built for developers. Режим доступа: https://github.com/ bruneli/scalaopt.

13. Fritsch F.N., Carlson R.E. Monotone piecewise cubic interpolation // SIAM Journal of Numerical Analysis. 1980. Vol. 17. No. 2. Pp. 238-246.

14. DuPont refrigerant expert. Cycle calculation program // Chemours. Режим доступа: https://www.chemours. com/Refrigerants/en US/products/DUPREX/DUPREX.html.

15. Yana Motta S.F., Sethi A., Vera Becera E.D. et al. Genetron properties. Режим доступа: https://www.honeywell-refrigerants.com/americas/resources/?cat=app

16. NIST reference fluid thermodynamic and transport properties database (REFPROP). Режим доступа: http:// www.nist.gov/srd/nist23.cfm.

17. Coquelet C., El Abbadi J., Houriez C. Prediction of thermodynamic properties of refrigerant fluids with a new three-parameter cubic equation of state // International Journal of Refrigeration. 2016. Vol. 69. Pp. 418-436.

18. Poling B.E., Prausnitz J.M., O'Connell J.P. Properties of gases and liquids. Fifth edition. McGRAW-HILL, 2001.

19. Mulero A., Cachadina I., Tian J. Ideal gas contribution to the isobaric heat capacity of refrigerants: Polling et al.'s polynomial correlation vs. DIPPR data // Journal of Chemical Thermodynamics. 2013. Vol. 61. Pp. 90-99.

20. Гершкович Г.П. Кое-что из американского опыта проектирования тепловых насосов // Сантехника, отопление, кондиционирование. 2008. № 9. Режим доступа: https:/www.c-o-k.ru/articles/koechto-izamerikanskogo-opyta-proektirovaniya-teplovyh-nasosov.

21. Тимофеев Д.В., Малявина Е.Г. Разработка численной модели теплопередачи между грунтом и термоскважиной // Вестник гражданских инженеров. 2015. № 5 (52). С. 196-202.

О б а в торах: Тимофеев Даниил Викторович - аспирант кафедры отопления и вентиляции, Национальный исследовательский Московский государственный строительный университет (НИУ МГСУ), 129337, г. Москва, Ярославское шоссе, д. 26, daniil@fastmail.fm;

Малявина Елена Георгиевна - кандидат технических наук, профессор, профессор кафедры теплогазоснабжения и вентиляции, Национальный исследовательский университет, Московский государственный строительный университет (НИУ МГСУ), 129337, г. Москва, Ярославское шоссе, д. 26, emal@list.ru. 


\section{REFERENCES}

1. Chen Y., Halm N.P., Groll E.A. et al. Mathematical modeling of scroll compressors - part I: compression process modeling. International Journal of Refrigeration. 2002, vol. 25 , no. 6 , pp. $731-750$.

2. Duvanov S.A., Il'in A.K. Metod modelirovaniya teplovykh nasosov [Method for Modeling the Operation of Heat Pumps]. Vestnik AGTU [Bulletin of the Astrakhan State Technical University]. 2005, issue 2 (25), pp. 66-70. (In Russian)

3. Allien J.J., Hamilton J.F. Steady-state Reciprocating Water Chiller Models. ASHRAE Transactions. 1983, 89 (2A), pp. 398-407.

4. Huchtemann K., Dirk M. Advanced Simulation Methods for Heat Pump Systems. Proceedings of the 7th Modelica Conference. Como, Italy. 2009, Sep 20-22. Pp. 798-803.

5. Kinab E., Marchio D., Riviere P. et al. Reversible Heat Pump Model for Seasonal Performance Optimizations. Energy and Buildings. 2010, 42, pp. 2269-2280.

6. Jin H., Spitler J. A parameter Estimation based Model of Water to Water Heat Pumps for Use in Energy Calculation Programs. ASHRAE Transactions. 2002, 108 (1), pp. 3-17.

7. Zakula T. Heat Pump Simulation Model and Optimal Variable-speed Control for a Wide Range of Cooling Conditions : Master of Science in Building Technology Thesis. Massachusetts Institute of Technology, 2010.

8. Kavanaugh S., Rafferty K. Geothermal Heating and Cooling. Design of Ground-Source Heat Pump Systems. Atlanta, ASHRAE, 2014. 420 p.

9. Duprez M.-E., Dumont E., Frere M. Modeling of Reciprocating and Scroll Compressors. International Journal of Refrigeration. 2007, 30, pp. 873-886.

10. Duprez M.-E., Dumont E., Frere M. Modeling of Scroll Compressors - Improvements. International Journal of Refrigeration. 2010, 33, pp. 721-728.

11. Winandy E., Saavedra C., Lebrun J. Experimental Analysis and Simplified Modeling of a Hermetic Scroll Refrigeration Compressor. Applied Thermal Engeneering. 2001, 22, pp. 107-120.

Received in August 2016.

Adopted in revised form in October 2016.

Approved for publication in February 2017.
12. Scalaopt. Scala Numerical Optimization Library. Built for Developers. Available at: https://github.com/bruneli/ scalaopt.

13. Fritsch F.N., Carlson R.E. Monotone Piecewise $\mathrm{Cu}-$ bic Interpolation. SIAM Journal of Numerical Analysis. 1980, vol. 17 , no. 2, pp. 238-246.

14. DuPont Refrigerant Expert. Cycle Calculation Program. Chemours. Available at: https://www.chemours.com/ Refrigerants/en_US/products/DUPREX/DUPREX.html.

15. Yana Motta S.F., Sethi A., Vera Becera E.D. et al. Genetron Properties. Available at: https://www.honeywellrefrigerants.com/americas/resources/?cat=app.

16. NIST Reference Fluid Thermodynamic and Transport Properties Database (REFPROP). Available at: http:// www.nist.gov/srd/nist23.cfm.

17. Coquelet C., El Abbadi J., Houriez C. Prediction of Thermodynamic Properties of Refrigerant Fluids with a New Three-Parameter Cubic Equation of State. International Journal of Refrigeration. 2016, 69, pp. 418-436.

18. Poling B.E., Prausnitz J.M., O’Connell J.P. Properties of Gases and Liquids. Fifth edition. McGRAW-HILL, 2001.

19. Mulero A., Cachadina I., Tian J. Ideal Gas Contribution to the Isobaric Heat Capacity Of Refrigerants: Polling et al.'S Polynomial Correlation vs. DIPPR data. Journal of Chemical Thermodynamics. 2013, 61, pp. 90-99.

20. Gershkovich G.P. Koye-chto iz amerikanskogo opyta proyektirovaniya teplovykh nasosov [Something from the American Experience in Designing Heat Pumps]. Santekhnika, otopleniye, konditsionirovaniye [Plumbing, Heating, Air Conditioning]. 2008, no. 9. Available at: https://www.c-o-k. $\mathrm{ru} /$ articles/koechto-iz-amerikanskogo-opyta-proektirovaniyateplovyh-nasosov. (In Russian)

21. Timofeyev D.V., Malyavina Ye.G. Razrabotka chislennoy modeli teploperedachi mezhdu gruntom i termoskvazhinoy [Development of the Numerical Model of the Heat Transfer between the Soil and the Thermowave]. Vestnik grazhdanskikh inzhenerov [Civil Engineers Bulletin]. 2015, no. 5 (52), pp. 196-202. (In Russian)

About the authors: Timofeev Daniil Victorovich - Postgraduate Student of Department Heating and Ventilation, Moscow State University of Civil Engineering (National Research University) (MGSU), 26 Yaroslavskoe shosse, Moscow, 129337, Russian Federation, daniil@fastmail.fm;

Malyavina Elena Georgievna - Candidate of Technical Science, Professor, Professor of Department Heating and Ventilation, Moscow State University of Civil Engineering (National Research University) (MGSU), 26 Yaroslavskoe shosse, Moscow, 129337, Russian Federation, emal@list.ru. 